ИЗВЕСТИЯ АКАДЕМИИ НАУК ЭСТОНСКОИ ССР. ТОМ 26 ФИЗИКА * МАТЕМАТИКА, 1977, № 1

\title{
О ФУРЬЕ-СПЕКТРАХ ЯМР ЖИДКОСТЕЙ В ЭКСПЕРИМЕНТАХ спин-лоКинг
}

Времена поперечной релаксации отдельных спектральных линий в сложном спектре ЯМР могут быть определены из реакции спин-системы на последовательность импульсов Карра-Парселла $\left[{ }^{1,2}\right]$. Для этой же цели используют и т. н. эксперименты спин-локинг $\left[{ }^{3}\right]$. Последние позволяют определять также скорости химического обмена $[4,5]$, различия в химическом сдвиге и во времени жизни обменивающихся ядер в разных положениях [ $\left.{ }^{6}\right]$ и константы спин-спиновой связи между резонансным и быстро релаксирующим нерезонансным ядрами [7].

Подготовка состояния спин-системы к спин-локингу может осуществляться двумя способами: применением адиабатического полупрохождения $\left[{ }^{4,5,8}\right]$ или применением 90 -градусного импульса $\left[{ }^{3,9}\right]$. При интерпретации результатов эксперимента, как правило, предполагается экспоненциальное уменьшение намагниченности с постоянной времени $T_{1 \rho}$ во время спин-локинга. Во многих случаях $T_{1 \rho}$ принимается равным $T_{2}$.

В настоящей статье на основе кинетического уравнения ВангснессаБлоха-Редфильда (ВБР) теоретически изучаются спектры жидкостей в экспериментах спин-локинг. Применением метода супероператоров $\left[{ }^{10}\right]$ получено формальное решение проблемы в случае импульсной подготовки состояния спин-системы. Выведенные общие формулы используются для изучения зависимости амплитуд спектральных линий от длительности спин-локинга в системе $A X$ с внутримолекулярным диполь-дипольным механизмом релаксации. Анализируются два варианта эксперимента спин-локинг: 1) ВЧ поле действует непосредственно на переходы обоих ядер и 2) ВЧ поле действует непосредственно на переходы только одного ядра.

\section{1. Описание эксперимента и определение состояния спин-системы}

Рассматриваемый эксперимент проводится по следующей схеме: в момент $t=0$ спин-система, находящаяся при равновесной поляризации в постоянном магнитном поле (z-направление), подвергается воздействию ВЧ импульса продолжительностью $\boldsymbol{\tau}$ с вектором $\mathbf{H}_{1}$, направленным по оси $x$. В момент окончания первого импульса на спин-систему накладывается второй ВЧ импульс продолжительностью $T$ с вектором $\mathbf{H}_{2}$, направленным по оси $y$ (спин-локинг). Частотный спектр получается Фурье-преобразованием напряжения, индуцируемого ядерной намагни- 
ченностью в приемной катушке после окончания второго импульса. В это время спин-система находится в постоянном магнитном поле, а приемная катушка идет по оси $y$. Следовательно, для определения параметров наблюдаемого спектра необходимо знать изменение состояния спин-системы во времени с момента окончания второго импульса.

Для описания состояния спин-системы используется, как обычно, оператор плотности $\sigma(t)$ и предполагается, что равновесное состояние в постоянном магнитном поле описывается оператором $\sigma_{0}$ в высокотемпературном приближении $\left[{ }^{10}\right]$. Девиация $\chi$ оператора плотности $\sigma$ от равновесного $\sigma_{0}$ определяется из кинетического уравнения ВБР, преобразованного во вращающиеся с частотой ВЧ полей $\omega_{1}$ координаты. Величины во вращающейся координатной системе отмечаются индек$\operatorname{com} T$.

Во время регистрации сигнала, т. е. с момента окончания второго импульса, изменение девиации $\chi_{T}$ подчиняется уравнению

$$
\dot{\chi}_{T}=-\left(\mathrm{i} \Omega_{0}-\Re\right) \chi_{T} .
$$

Здесь ее начальным состоянием является, естественно, состояние спинсистемы в момент окончания второго импульса. Во время воздействия второго импульса девиация изменяется согласно уравнению

$$
\dot{\chi}_{T}=-\left(\mathrm{i} \Omega_{2}-\Re\right) \chi_{T}-\mathrm{i} \Omega_{2} \sigma_{0},
$$

где ее начальным значением является девиация в момент окончания первого импульса. Изменение девиации во время воздействия первого импульса описывается уравнением

$$
\dot{\chi}_{T}=-\mathrm{i} \Omega_{1} \chi_{T}-\mathrm{i} \Omega_{1} \sigma_{0},
$$

при этом ее начальным состоянием служит равновесное состояние $\sigma_{0}$ в постоянном магнитном поле.

Супероператоры $\mathfrak{R}_{k}$ в уравнениях (1)-(3) учитывают влияние внешних магнитных полей на состояние спин-системы

Здесь

$$
\mathfrak{\Omega}_{k} \mathbf{Q}=\left[\mathbf{H}_{T}+\mathbf{H}_{k T}, \mathbf{Q}\right], \quad k=0,1,2,
$$

$$
\begin{aligned}
& \mathbf{H}_{T}=\mathbf{H}_{0}+\omega_{1} \sum_{i} \mathbf{I}_{z}(i), \\
& \mathbf{H}_{k T}=\sum_{i} h_{k i}\left[\delta_{k 1} \mathbf{I}_{x}(i)+\delta_{k 2} \mathbf{I}_{y}(i)\right], \\
& h_{k i}=-\gamma_{i} H_{k},
\end{aligned}
$$

$\mathbf{H}_{0}$ - гамильтониан спин-системы в постоянном магнитном поле, $H_{k}$ $(k=1,2)$ - амплитуды ВЧ полей первого и второго импульсов, а I $(i)$ и $\gamma_{i}$ имеют общепринятый смысл. Влияние релаксационных процессов учитывается супероператором $\Re$, компонентами которого являются редфильдовские релаксационные коэффициенты $\Re_{a b c d}\left[{ }^{11}\right]$. В условиях сильного сужения значения релаксационных коэффициентов в лабораторной и во вращающейся системах координат совпадают $\left[{ }^{12}\right]$.

Использование кинетического уравнения в форме (3) оправдано только тогда, когда изменение состояния спин-системы во время воздействия первого импульса обусловлено влиянием в основном внешних полей. Релаксационными процессами можно пренебречь при выполнении условия

$$
\left|h_{1 i}\right|, \quad \frac{1}{\tau} \gg\left|\Re_{a b c d}\right| .
$$

Формальное решение уравнений (1)-(3) приводит к следующей за- 
висимости состояния спин-системы от времени в интервале регистрации:

$$
\chi_{T}(t)=\mathrm{e}^{-t\left(\mathrm{i}_{0}-\Re\right)} \chi_{T}^{(0)}, \quad t \geqslant 0 .
$$

В последнем соотношении за начало отсчета времени выбран момент конца спин-локинга, $\chi_{T}^{(0)}-$ значение девиации в этот момент

$\chi_{T}^{(0)}=\left\{\mathrm{e}^{-T\left(\mathrm{i} \Omega_{2}-\Re\right)}\left[\mathrm{e}^{-\mathrm{i} \tau \Omega_{1}}+\left(\mathrm{i} \Omega_{2}-\Re\right)^{-1} \mathrm{i} \Omega_{2}-\mathcal{E}\right]-\left(\mathrm{i} \Omega_{2}-\Re\right)^{-1} \mathrm{i} \Omega_{2}\right\} \sigma_{0}$,

а $\mathcal{E}$ является единичным супероператором.

Выражения (9) и (10) вместе с $\sigma_{0}$ определяют состояние спин-системы и позволяют тем самым, в принципе, вычислять наблюдаемый частотный спектр.

\section{2. О частотном спектре}

В рассматриваемом эксперименте наблюдаемый частотный спектр $S(\omega)$ получается Фурье-преобразованием сигнала, индуцируемого в приемной катушке, направленной по оси $y$ лабораторной системы координат. Используя т. н. диагональный базис $|k\rangle,|l\rangle, \ldots\left[{ }^{10}\right]$

$$
\left[-\mathrm{i}\left(\Omega_{0}-\omega_{1} \varepsilon\right)+\Re\right]_{k l m n}=\left(r_{k l}+\mathrm{i} \Omega_{k l}\right) \delta_{k m} \delta_{l n}
$$

и определенный выше оператор плотности, можно представить частотный спектр в виде суперпозиции линий лоренцевой формы

$$
\begin{gathered}
\sim-\sum_{k, l} I_{k l}\left\{\frac{r_{h l}}{r_{k l}^{2}+\left(\Omega_{k l}-\omega\right)^{2}} \cos \left(\varphi_{k l}+\varphi\right)-\frac{\Omega_{k l}-\omega}{r_{k l}^{2}+\left(\Omega_{k l}-\omega\right)^{2}} \sin \left(\varphi_{k l}+\varphi\right)\right\}- \\
-\mathrm{i} \sum_{k, l} I_{k l}\left\{\frac{\Omega_{k l}-\omega}{r_{k l}^{2}+\left(\Omega_{k l} \div \omega\right)^{2}} \cos \left(\varphi_{k l}+\varphi\right)+\frac{r_{k l}}{r_{k l}^{2}+\left(\Omega_{k l}-\omega\right)^{2}} \sin \left(\varphi_{k l}+\varphi\right)\right\} \cdot(12)
\end{gathered}
$$

Здесь

$$
\begin{gathered}
I_{k l}=\left\{\left[r_{k l} \operatorname{Re}\left(\mathrm{F}_{-l k} \chi_{T k l}^{(0)}\right)-\Omega_{k l} \operatorname{Im}\left(\mathrm{F}_{-l k} \chi_{T k l}^{(0)}\right)\right]^{2}+\right. \\
\left.+\left[r_{k l} \operatorname{Im}\left(\mathbf{F}_{-l k} \chi_{T k l}^{(0)}\right)+\Omega_{k l} \operatorname{Re}\left(\mathbf{F}_{-l k} \chi_{T k l}^{(0)}\right)\right]^{2}\right\}^{1 / 2} \\
\varphi_{k l}=\arctan \frac{r_{k l} \operatorname{Re}\left(\mathbf{F}_{-l k} \chi_{T k l}^{(0)}\right)-\Omega_{k l} \operatorname{Im}\left(\mathbf{F}_{-l k} \chi_{T h l}^{(0)}\right)}{r_{k l} \operatorname{Im}\left(\mathbf{F}_{-l k} \chi_{T k l}^{(0)}\right)+\Omega_{k l} \operatorname{Re}\left(\mathbf{F}_{-l k} \chi_{T k l}^{(0)}\right)}, \\
\mathbf{F}_{ \pm}=\sum_{i} \gamma_{i} \mathbf{I}_{ \pm}(i) .
\end{gathered}
$$

Следовательно, каждой паре уровней диагонального базиса, в общем, соответствует в спектре один элемент, который является суммой лоренцевых поглощенноподобного и дисперсионноподобного слагаемых. Действительное число элементов в спектре определяется числом ненулевых элементов оператора $\mathbf{F}_{-}$в диагональном базисе. Изменением фазы $\varphi$ любую линию в спектре можно преобразовать к виду чистой линии поглощения или дисперсии. В случае определенного значения $\varphi$ только одна линия имеет правильную форму.

Если постоянное магнитное поле достаточно сильно и спектр собственных значений $\mathbf{H}_{0}$ лишен близких уровней, то в первом приближении диагональный базис будет совпадать с базисом из собственных функций $\mathbf{H}_{0}$ (а-базисом) $\left[{ }^{10}\right]$, т. е. мы можем записать 


$$
\begin{aligned}
& r_{k l}=\Re_{a b a b}=-\frac{1}{T_{2 a b}}, \\
& \Omega_{k l}=\omega_{a b} .
\end{aligned}
$$

В этом случае число линий в спектре, а также положение их центров и естественные ширины совпадают с таковыми спектра медленного прохождения со слабым ВЧ полем. Вместе с тем интенсивности и амплитуды линий в спектре спин-локинга сложным образом зависят от параметров обоих импульсов, от параметров спин-системы и от механизма релаксации. Для определения амплитуд (и интенсивностей) линий требуется вычисление девиации $\chi_{T}^{(0)}$ из уравнения (10). Это вычисление намного упрощается, если оправдано приближение

что приводит к выражению

$$
\left(i \Omega_{2}-\Re\right)^{-1} \mathrm{i} \Omega_{2}=\varepsilon,
$$

$$
\chi_{T}^{0}=\left[\mathrm{e}^{-T\left(\mathrm{i} \Omega_{2}-\Re\right)} \mathrm{e}^{-\mathrm{i} \tau \Omega_{1}}-\varepsilon\right] \sigma_{0} .
$$

\section{3. Система $\boldsymbol{A X}$}

В случае использования эксперимента спин-локинг для измерения времен релаксации необходимо теоретически предсказать, как зависят амплитуды спектральных линий от длительности интервала спин-локинга (от остальных параметров импульсов) и от релаксационных параметров. На основе выражений (12), (13) и (10) или (19) трудно установить характер этой зависимости. Во всяком случае нет никаких оснований считать ее простой.

Рассмотрим эту зависимость для системы $A X$ с учетом лишь механизма внутримолекулярной диполь-дипольной релаксации. Анализ проведем аналогично изучению системы $A X$ в $\left[{ }^{13}\right]$, т. е. будем считать, что $a$-базис - диагональный. Матричные элементы операторов в этом базисе обозначаются как $\mathbf{Q}_{a, b}$, где $a, b=1,2,3,4$ соответствуют мультипликативным собственным функциям $|\alpha \alpha\rangle,|\alpha \beta\rangle,|\beta \alpha\rangle$ и $|\beta \beta\rangle$. B $\left[{ }^{13}\right]$ показано, что амплитуды спектральных линий зависят от следующих матричных элементов девиационного оператора: $\chi_{T 1,3}^{(0)}, \chi_{T 2,4}^{(0)}$ $(A$-линии $)$ и $\chi_{T 1,2}^{(0)}, \chi_{T 3,4}^{(0)}(X$-линии $)$. На основе $(16)$ и $(17)$ в выражениях (13) и (14) будем пренебрегать членами, пропорциональными $\Re_{a b c d}$. Оператор $\chi_{T}^{(0)}$ определим путем разложения операторов и супероператоров по т. н. симметричному базису $\left[{ }^{13}\right]$, а в целях сокращения выкладок воспользуемся выражением (19). (Необходимые элементы $\chi_{T}^{(0)}$ в $a$-базисе выражены через элементы $\chi_{T}^{(0)}$ симметричного базиса в $\left[{ }^{13}\right]$.) При этом необходимо знание матриц супероператоров $\mathrm{e}^{\alpha \Omega} \quad\left(\Re=\mathrm{i} \Omega_{2}-\Re, \mathrm{i} \Omega_{1}\right)$. Матрица супероператора $\Re\left(\right.$ и оператора $\left.\sigma_{0}\right)$ в симметричном базисе приведена в [13], а матрицы $\Omega_{1}$ и $\Omega_{2}$ нетрудно установить с помощью определений (4) (см. приложение). Для избежания трудностей, связанных с нахождением суммы бесконечного ряда каждого матричного элемента $\mathrm{e}^{\alpha \Re}$ путем прямого применения матриц $\Omega_{1}, \Omega_{2}$ и $\Re$, матрицу е ${ }^{\alpha \Re}$ будем определять через диагональную в симметричном базисе матрицу $\Re_{\mathfrak{D}}\left[{ }^{13}\right]$. В настоящей статье из-за отсутствия точного диагонализирующего преобразования $\mathfrak{D}, \mathfrak{D}^{-1}$ использованы приближенные значения $\mathfrak{I}_{\mathfrak{D}}$, $\mathfrak{D}$ и $\mathfrak{D}^{-1}$. Поскольку последние зависят и от соотношений между величинами в $\Re$, следует рассмотреть разные варианты эксперимента. 
Н ес електи в ны й с пин - локинг. Пусть оба импульса неселективны в смысле

$$
\left|h_{k i}\right| \gg\left|\delta_{i}\right|,|I|,\left|\frac{1}{T_{n}}\right|, \quad k=1,2 ; i=A, X ; n=1,2, \ldots, 5 .
$$

Здесь приближенное значение $\Omega_{1 D}$ определяется по аналогии с соответствующим значением в $\left[{ }^{13}\right]$ : во-первых, диагонализируем $\Omega_{1}$ относительно $h_{1 i}$ и на основе (20) пренебрегаем недиагональными элементами в местах, где разность диагональных элементов порядка $\left|h_{k i}\right|$. Во-вторых, диагонализируем полученную приближенную матрицу и используем ее для получения приближенной матрицы ехр $\left(-\mathrm{i} \tau \Omega_{1}\right)$. Интересно отметить, что вследствие приближения отпадает зависимость ехр (-i $\left.\Omega_{1}\right)$ от $\delta_{i}$.

Процедура получения приближенной матрицы $\exp \left[-T\left(\mathrm{i} \Omega_{2}-\Re\right)\right]$ отличается от описанной выше незначительно. Сначала симметризуем матрицу $\mathrm{i} \Omega_{2}-\Re$, затем диагонализируем ее относительно $h_{2 i}$ и на основе (20) проводим первое приближение. Далее, полученную матрицу диагонализируем относительно $I$ и проводим второе приближение - пренебрегаем недиагональными элементами (величина их порядка $\left.\left|1 / T_{n}\right|\right)$ в местах, где разность диагональных элементов порядка $l_{2}$,

$$
l_{k}=\frac{1}{2} \sqrt{\left(h_{k A}-h_{k X}\right)^{2}+\frac{1}{4} I^{2},} \quad k=1,2 .
$$

Второе приближение гарантируется хорошей разрешенностью линий в спектре системы $A X$. Наконец, диагонализируем оставшуюся матрицу и с ее помощью определяем приближенное значение $\exp \left[-T\left(\mathrm{i}_{2}-\Re\right)\right]$. При этом $\mathscr{D}$ и $\mathfrak{D}^{-1}$ содержат четыре различных преобразования. Здесь также отпадает зависимость от $\delta_{i}$.

Оказывается, что амплитуды (и фазы) наблюдаемых спектральных линий сложным образом зависят от длительности интервала $T$. Эта зависимость различна для разных линий и содержит, наряду с параметрами обоих импульсов, все релаксационные коэффициенты $1 / T_{n}$.

Если первый импульс 90-градусный,

$$
\gamma=\tau_{1} h_{1}=\pi / 2 ; \quad h_{k}=\frac{1}{2}\left(h_{k A}+h_{k X}\right), \quad k=1,2,
$$

то амплитуды всех линий зависят от интервала спин-локинга $T$ экспоненциально с постоянной времени

$$
\begin{gathered}
\frac{1}{T_{2}}\left(\frac{1}{T_{2}}=-\Re_{1212}=-\Re_{1313}=-\Re_{2424}=-\Re_{3434}\right), \text { т. е. } \\
I_{a b}(T) \sim \mathrm{e}^{-\frac{1}{T_{2}} T}
\end{gathered}
$$

Отметим, что для выполнения (23) наряду с (22) должны выполняться следующие три условия:

$$
\frac{1}{\tau} \gg\left|l_{1}\right| ; \quad\left|h_{k A}-h_{k X}\right| \ll\left|\frac{1}{2} I\right|, \quad k=1,2 .
$$

Если первый импульс не является 90-градусным, имеем

$$
I_{a b}(T) \sim \mathrm{e}^{-\frac{1}{T_{2}} T}\left[\sin ^{2} \gamma+\mathrm{e}^{-\Theta_{1} T} \sin h_{2} T\left(\mathrm{e}^{-\Theta_{1} T} \sin h_{2} T \cos ^{2} \gamma \mp\right.\right.
$$




$$
\begin{gathered}
\left.\left.\mp \sin \ell_{2} T \sin 2 \gamma\right)\right]^{1 / 2}, \\
\varphi_{a b}=\arctan \left[-\frac{\sin \gamma \mp \mathrm{e}^{-\Theta_{1} T} \sin h_{2} T \sin l_{2} T \cos \gamma}{\mathrm{e}^{-\Theta_{1} T} \sin h_{2} T \cos l_{2} T \cos \gamma}\right] .
\end{gathered}
$$

Здесь верхние знаки относятся к случаю $a=1$ и

$$
\Theta_{1}=\frac{7}{34} \frac{1}{T_{2}}
$$

Если первый импульс мало отличается от 90-градусного,

$$
\gamma=\frac{\pi}{2}+\alpha, \quad|\alpha| \ll 1
$$

и учитывается только первое приближение по $\alpha$, то

$$
I_{a b}(T) \sim \mathrm{e}^{-\frac{1}{T_{2}} T} \quad\left(1 \pm \mathrm{e}^{-\Theta_{1} T} \alpha \sin h_{2} T \sin l_{2} T\right) .
$$

С ел ек ти вны й спин-локинг. Рассмотрим эксперимент, в котором второй импульс непосредственно воздействует на переходы только ядра $X$,

$$
\left|\delta_{A}\right| \gg\left|h_{2 i}\right| \gg\left|\delta_{X}\right|,|I|,\left|\frac{1}{T_{n}}\right|, i=A, X ; \quad n=1,2, \ldots, 5 .
$$

Приближенная матрица $\exp \left[-T\left(\mathrm{i}_{2}-\Re\right)\right]$ определяется аналогично предыдущему случаю. После симметризации $\mathrm{i} \Omega_{2}-\Re$ диагонализируем ее относительно $\delta_{A}$ и проводим первое приближение. На этом этапе отпадает зависимость от $h_{2 A}$. Полученную матрицу диагонализируем относительно $h_{2 x}$ и проводим второе приближение. В результате этого отпадает зависимость приближенного $\exp \left[-T\left(\mathrm{i} \Omega_{2}-\Re\right)\right]$ от $\delta_{X}, J$ и $1 / T_{4}$.

В принципе первый импульс может быть и неселективным, т. е. может удовлетворяться условие (20). В этом случае матрица ехр (-i $\left.\tau \Omega_{1}\right)$, будет, естественно, совпадать с матрицей, определенной выше. Селективный подготовительный импульс удовлетворяет условию типа (29) и потому определение матрицы, описывающей его влияние на состояние спин-системы, аналогично использованному при определении матрицы $\exp \left[-T\left(\mathrm{i} \Omega_{2}-\Re\right)\right]$.

Оба способа подготовки спин-системы к спин-локингу различаются лишь тем, что при использовании первого в спектре могут появиться наряду с $X$-линиями еще две $A$-линии, а при использовании второго нет. Діля $X$-линии в обоих случаях получается

$$
\begin{gathered}
I_{a b}(T) \sim \mathrm{e}^{-\frac{1}{T_{2}} T}\left[\sin ^{2} \gamma+\mathrm{e}^{-2 \Theta_{2} T} \sin ^{2} h_{2 X} T \cos ^{2} \gamma\right]^{1 / 2}, \\
\varphi_{a b}=\arctan \left(-\frac{\sin \gamma}{\mathrm{e}^{-\Theta_{2} T} \sin h_{2 X} T \cos \gamma}\right),
\end{gathered}
$$

где

$$
\Theta_{2}=\frac{3}{34} \frac{1}{T_{2}} .
$$

При точном 90-градусном первом импульсе $X$-линии зависят от интервала $T$ экспоненциально. Зависимость экспоненциальна также в первом приближении по $\alpha$ при малом отклонении от $90^{\circ}$. 


\section{Заключение}

Теоретически изучены Фурье-спектры жидкостей в экспериментах спин-локинг в случае импульсной подготовки состояния спин-системы.

Выяснено, что при достаточно сильном постоянном магнитном поле центры и параметры полуширины линий в спектрах спин-локинг совпадают с соответствующими величинами в спектре медленного прохождения при условии отсутствия перекрывания линий в последнем. Линии спектра спин-локинг являются суммами лоренцевых поглощенноподобных и дисперсионноподобных компонентов. Изменением фазы только одна линия может быть приведена к чистой форме поглощения или дисперсии одновременно.

Формулы (10) и (13) не позволяют, в общем, предполагать простую экспоненциальную зависимость амплитуд спектральных линий от длительности интервала спин-локинг. Все же в системе $A X$ с внутримолекулярным диполь-дипольным механизмом релаксации эта зависимость экспоненциальна, если только подготовительный импульс является 90градусным. В противном случае вышеотмеченная зависимость содержит один или два гармонически модулированных экспоненциальных члена, временная константа которых отличается от $1 / T_{2}$ в пределах $10-20 \%$.

Алгебраическое определение зависимости амплитуд линий от длительности интервала спин-локинг из основных выражений (10) и (13) связано с трудоемкими вычислениями и нет уверенности в том, что оно вообе выполнимо без привлечения многократных приближений.

\section{Приложение}

1. Матричные элементы супероператора $\mathfrak{L}_{1}$ в симметричном базисе:

$$
\begin{aligned}
& \mathfrak{S}_{1 k l}=\mathfrak{Q}_{1 l k}, \\
& \Omega_{1 k l}=\left\{\begin{array}{cllll}
-h_{1 A}, & (k, l)=(1,3), & (5,8), & (7,11), & (10,12) . \\
\delta_{A}, & (k, l)=(2,3), & (4,5), & (6,7), & (9,10) . \\
\frac{1}{2} I, & (k, l)=(2,5), & (3,4), & (11,14), & (12,13) . \\
\delta_{X}, & (k, l)=(6,9), & (7,10), & (11,12), & (13,14) . \\
-h_{1 X}, & (k, l)=(4,6), & (5,7), & (8,11), & (13,15) . \\
0, & \text { в остальных случаях } k \leqslant 1 . &
\end{array}\right.
\end{aligned}
$$

Здесь

$$
I=2 \pi J ; \quad \delta_{i}=\omega_{0 i}+\omega_{1} ; \quad i=A, X .
$$

2. Матричные элементы супероператора $\Omega_{2}$ в симметричном базисе. Матрица супероператора $\Omega_{2}$ получается из матрицы $\mathfrak{\Omega}_{1}$, если в последней принять $h_{1 A}=h_{1 X}=0$ и прибавить к ней матрицу $\mathfrak{\Omega}_{2}^{\prime}$ со следующими ненулевыми элементами:

$$
\mathfrak{S}_{2 k l}^{\prime}=\left\{\begin{array}{rlll}
-i h_{2 A}, & (k, l)=(1,2) . & \\
i h_{2 A}, & (k, l)=(4,8), \quad(6,11), & (9,12) . \\
-i h_{2 X}, & (k, l)=(4,9), & (5,10), & (8,12) . \\
i h_{2 X}, & (k, l)=(14,15) . & \\
\mathfrak{S}_{2 k l}^{\prime}=-\mathfrak{S}_{2 l k}^{\prime} . &
\end{array}\right.
$$




\section{ЛИТЕ РАТ У Р А}

1. Vold R. L., J. Chem. Phys., 56, 3210 (1972).

2. Vold R. L., Vold R. R., Simo n H. E., J. Magn. Resonance, 11, 283 (1973).

3. Sol o m o n I., C. R. Acad. Sci., 248, 92 (1959).

4. Me i b o o m S., J. Chem. Phys., 34, 375 (1961).

5. S y ke s B. D., J. Amer. Chem. Soc., 91, 949 (1969).

6. Deverel1 C., Morgan R. E., Strange J. H., Mol. Phys., 18, 553 (1970).

7. Morg a n R. E., Strange J. H., Mol. Phys., 17, 397 (1969).

8. Neely J. W., Krugh T. R., J. Magn. Resonance, 9, 84 (1973).

9. Freem a n R., Hill H. D. W., J. Chem. Phys., 55, 1985 (1971).

10. К унд л а Э., Изв. АН ЭССР, Физ. Матем., 22, 267 (1973).

11. Redfield A. G., Advances in Magnetic Resonance, 1, Academic Press, N. Y., 1966.

12. Nageswara Rao B. D., Phys. Rev., 137, A467 (1965).

13. К ундл а Э., Изв. АН ЭССР, Физ. Матем., 23, 396 (1974).

\section{Институт кибернетики} Академии наук Эстонской ССР
Поступила в редакцию 26/IV 1976

\section{E. KUNDLA}

\section{VEDELIKE TMR FOURIER' SPEKTRITEST SPIN-LOCKING-KATSES}

On teoreetiliselt uuritud nn. spin-locking-katses impulssettevalmistuse puhul saadavaid TMR Fourier' spektreid. Ilmnes, et üldiselt pole spektraaljoonte amplituudide sõltuvus spin-locking'i intervallist eksponentsiaalne, küll aga on see nii sisemolekulaarse dipool-dipoolse relaksatsioonimehhanismiga AX-süsteemi puhul, kui ettevalmistav impulss on $90^{\circ}$. Teistsuguse impulsi korral sisaldab see sõltuvus mitu harmooniliselt moduleeritud eksponentsiaalset liiget. Viimaste ajakonstandid erinevad $1 / T_{2}$-st.

\section{E. KUNDLA}

\section{ON NMR FOURIER SPECTRA OF LIQUIDS IN SPIN-LOCKING EXPERIMENTS}

The NMR Fourier spectra of liquids obtained in the spin-locking experiments with pulse preparing are investigated theoretically.

It appears that the line amplitudes do not depend, in general, on the spin-locking interval exponentially. In the cases of the $A X$ system with intramolecular dipole-dipole relaxation mechanism this dependence, however, is exponential only if the preparation pulse is a $90^{\circ}$ one. When the preparation pulse is not a $90^{\circ}$-pulse, then the abovementioned dependence contains several harmonically modulated exponential terms whose time constants differ from $1 / T_{2}$. 